\title{
Nucleic Acid Vaccine Targeting Nogo-66 Receptor and Paired Immunoglobulin-Like Receptor B as an Immunotherapy Strategy for Spinal Cord Injury in Rats
}

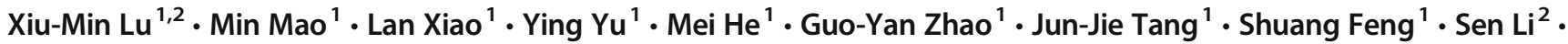 \\ Cheng-Ming $\mathrm{He}^{1} \cdot$ Yong-Tang Wang $^{2}$
}

Published online: 6 March 2019

(C) The American Society for Experimental NeuroTherapeutics, Inc. 2019

\begin{abstract}
Nogo-66 receptor (NgR) and paired immunoglobulin-like receptor B (PirB) are two common receptors of various myelinassociated inhibitors (MAIs) and, thus, play an important role in MAIs-induced inhibitory signalling of regeneration following spinal cord injury (SCI). Based on the concept of protective autoimmunity, vaccine approaches could induce the production of antibodies against inhibitors in myelin, such as using purified myelin, spinal cord homogenates, or MAIs receptor NgR, in order to block the inhibitory effects and promote functional recovery in SCI models. However, due to the complication of the molecules and the mechanisms involved in MAIs-mediated inhibitory signalling, these immunotherapy strategies have yielded inconsistent outcomes. Therefore, we hypothesized that the choice and modification of self-antigens, and co-regulating multiple targets, may be more effective in repairing the injured spinal cord and improving functional recovery. In this study, $\mathrm{NgR}$ and PirB were selected to construct a double-targeted granulocyte-macrophage colony stimulating factor-NgR-PirB (GMCSF-NgR-PirB) nucleic acid vaccine, and investigate the efficacy of this immunotherapy in a spinal cord injury model in rats. The results showed that this vaccination could stimulate the production of antibodies against $\mathrm{NgR}$ and PirB, block the inhibitory effects mediated by various MAIs, and promote nerve regeneration and functional recovery after spinal cord injury. These findings suggest that nucleic acid vaccination against $\mathrm{NgR}$ and $\mathrm{PirB}$ can be a promising therapeutic strategy for $\mathrm{SCI}$ and other central nervous system diseases and injuries.
\end{abstract}

Key Words Nogo-66 receptor $(\mathrm{NgR}) \cdot$ Paired immunoglobulin-like receptor B (PirB) $\cdot$ Nucleic acid vaccine $\cdot$ Functional recovery Spinal cord injury

\section{Introduction}

Growing evidence indicates that the regeneration failure following central nervous system (CNS) injury in adults is not due to intrinsic properties of CNS neurons but to the neurite outgrowth inhibitors around the injured site associated with myelin-associated inhibitors (MAIs), such as Nogo-A, the

Yong-Tang Wang

wangytlu@163.com

1 College of Pharmacy and Bioengineering, Chongqing University of Technology, Chongqing 400054, China

2 State Key Laboratory of Trauma, Burns and Combined Injury, Institute of Surgery Research, Daping Hospital, Third Military Medical University, Chongqing 400042, China myelin-associated glycoprotein (MAG), and the oligodendrocyte-myelin glycoprotein (OMgp) [1-3]. Interestingly, these three structurally different inhibitors share two common receptors, Nogo-66 receptor $(\mathrm{NgR})$ and paired immunoglobulin-like receptor B (PirB) [4-8]. Previous studies have shown that each of the three inhibitors can interact in high affinity with $\mathrm{NgR}$ and transduce the inhibitory signal into neurons via a complex composed of $\mathrm{NgR}$ and its co-receptors, such as p75 neurotrophin receptor (p75NTR), Nogo receptor-interacting protein 1 (LINGO-1), and TROY [9-12]. However, besides NgR, PirB, also known as leukocyte immunoglobulin (Ig)-like receptor B2 (LILRB2), was recently discovered as another common receptor for various MAIs, and it has also been proposed to mediate nearly all MAIs-mediated axon growth inhibition [13]. As an important CNS injury, traumatic spinal cord 
injury ( $\mathrm{SCI}$ ) is one of the leading causes of disabilities in young adults. With the advances in the study of PirB, it has been found that PirB has obvious inhibitory effects on axon regeneration in spinal cord injury, and the same as $\mathrm{NgR}$, provides a new therapeutic target for treatment of spinal cord injury through promoting axonal regeneration and functional recovery [13-16]. PirB gene knockout produced an axonal regeneration effect to a certain extent even better than $\mathrm{NgR}$, showing that in the myelin inhibition, PirB function may be better than that of previously found $\mathrm{NgR}$. If $\mathrm{NgR}$ and PirB are knocked down at the same time, neuronal growth cone collapse induced by myelin can be completely reversed. Experiments in vitro also showed that NgR gene knockout can promote PirB gene knockout to reverse the inhibitory effect of myelin on axon regeneration [17]. The above research confirmed that, in addition to NgR, PirB is also an important receptor that mediated the inhibition of axonal regeneration of MAIs, suggesting that the treatment of spinal cord injury should focus on the synergistic effect of $\mathrm{NgR}$ and PirB in a future study.

In the past few years, vaccine approaches were initially developed that could induce the production of antibodies against inhibitors in myelin to block the inhibitory effects and promote functional recovery in SCI models by immunizing with purified myelin, spinal cord homogenates, or their receptor with the concept of protective autoimmunity formulated [18]. Protective autoimmunity refers to a benign autoimmune response that contributes to the maintenance and protection of injured neurons and the promotion of recovery after traumatic injury to CNS [18-22]. Recently, a therapeutic vaccination protocol targeting $\mathrm{NgR}$ has also been used. Both the recombinant DNA and protein vaccine targeting $\mathrm{NgR}$ were proven to promote axonal regeneration and functional recovery after SCI in adult rats [23-25]. However, there is still no therapeutic vaccination targeting PirB for the injured spinal cords, let alone the synergistic effect of $\mathrm{NgR}$ and PirB.

Therefore, we hypothesized that the production of antibodies against $\mathrm{NgR}$ and PirB may block the inhibitory effects mediated by various MAIs and promote nerve regeneration as well as functional recovery after spinal cord injury by immunizing with nucleic acid vaccine encoding $\mathrm{NgR}$ and PirB. To test this hypothesis, a recombinant plasmid, pcDNAGMCSF-NgR-PirB encoding human NgR, PirB and granulocyte-macrophage colony stimulating factor (GMCSF), an effective adjuvant molecular, was constructed. The recombinant plasmid then formed a composite nucleic acid vaccine with liposome, and the effects of the antisera on neurite outgrowth of human neuroblastoma SH-SY5Y cells, axonal regeneration and functional recovery in spinal cordinjured rats were further investigated, which may pave a way to the further research of CNS regeneration on the target of $\mathrm{NgR}$ and PirB.

\section{Materials and Methods}

\section{Animals}

Adult female Sprague-Dawley (SD) rats, provided by the Animal Breeding Center of the Institute of Surgery Research, Daping Hospital, Third Military Medical University, were housed in a light- and temperature-controlled room. All surgical interventions and postoperative animal care were carried out in accordance with the Guide for the Care and Use of Laboratory Animals (National Research Council, 1996, USA) and approved by the Animal Use and Care Committee of School of Medicine, Third Military Medical University.

\section{Construction, Identification and Expression Detection of Recombinant Eukaryotic Expression Vectors}

The DNA fragment of human GM-CSF, NgR and PirB was amplified, respectively, from clone vector pMD18-T, pGEM$\mathrm{T}$ and pGEM-T by using polymerase chain reaction (PCR) and subcloned into eukaryotic expression vector pcDNA3.1(+) in turn, and constructed pcDNA-GMCSF, pcDNA-NgR-PirB, and pcDNA-GMCSF-NgR-PirB expressing vectors as a single open reading frame, respectively. The recombinant plasmids pcDNA-GMCSF-NgR-PirB were amplified in $E$. coli DH5 $\alpha$ and identified by using PCR, enzyme digestion and sequencing, respectively, then were transfected into Chinese hamster ovary $(\mathrm{CHO})$ cells mediated by lipofectamine, and the expression of GMCSF-NgR-PirB fusion protein was detected after transfected 24 hours by using immunofluorescence and Western blot, respectively. The untouched CHO cells were used as blank control, and the transfected pcDNA3.1(+) plasmids as negative control.

\section{Preparation of Double Targeted GMCSF-NgR-PirB Nucleic Acid Vaccine and Immunization of Animals}

The nucleic acid vaccine was formed by mixing the recombinant plasmids pcDNA-GMCSF-NgR-PirB and liposome at the same volume and incubation for $20 \mathrm{~min}$ at room temperature. Five-week-old female SD rats were immunized with $100 \mu \mathrm{g}$ of the recombinant plasmid by injection into musculus tibialis bilaterally once weekly for 6 weeks. To compare the effect of immunotherapy of pcDNA-GMCSF-NgR-PirB (GMCSF-NgR-PirB IM) for spinal cord injury, the pcDNA3.1(+) (Blank IM), pcDNA-GMCSF (GMCSF IM), and pcDNA-NgR-PirB (NgR-PirB IM) vectors were used as controls, the rats untouched as normal control (Control), and rats unimmunized for negative control (SCI). Beginning in the first week after immunization, the animals were exsanguinated weekly for sera collection until the sixth week. The blood was collected and centrifuged at $3000 \mathrm{~g}$ for $30 \mathrm{~min}$ at room temperature for separating the antisera. The collected sera was 
used for ELISA analysis for detection of antibody response, and a total of 36 rats, six rats in each group, were then used for the following animal experiment.

\section{ELISA for Detection of Antibody Response}

ELISA analysis was used to detect serum antibody by using sera collected above as described previously [25]. Microtiter plates were coated with $100 \mu \mathrm{l}$ purified $\mathrm{hNgR}$ or hPirB (1 $\mu \mathrm{g} / \mathrm{ml}$ ) overnight at $4^{\circ} \mathrm{C}$. The plates were then incubated with rat sera and serially diluted with PBS for 2 hours at $37^{\circ} \mathrm{C}$. Bound antibody was reacted with HRP-conjugated goat antirat $\operatorname{IgG}\left(1: 10000\right.$; SABC, City, State) for 1 hour at $37^{\circ} \mathrm{C}$. Optical density (OD) at $450 \mathrm{~nm}$ was then measured with an ELISA reader (Thermo Fisher Scientific, USA), and the average antibody titers were shown as mean OD. Sera that produced net OD values greater than the mean OD plus $3 \mathrm{SD}$ obtained with a panel of pre-immune sera were considered to have significant antibody responses.

\section{Effects of Antisera on the Neurite Outgrowth of Human Neuroblastoma SH-SY5Y Cells}

Human neuroblastoma SH-SY5Y cells were considered as a reliable neuron model used to investigate neurite outgrowth. It has been shown that Nogo-A and its receptors, such as $\mathrm{NgR}$ and p75NTR are expressed in SH-SY5Y cells, and inhibit neurite outgrowth through their downstream signal RhoA/ ROCK2 pathway $[25,26]$. As one component of the $\mathrm{NgR}$ receptor complex, p75NTR has also been linked to the PirB signal transduction pathway. Therefore, PirB, another common receptor for MAIs, should also be expressed in SHSY5Y cells. In order to detect the effects of antisera on the neurite outgrowth, human neuroblastoma SH-SY5Y cells were used to investigate the neurite outgrowth as previously described [27]. The cells were cultured in Dulbecco's modified Eagle's medium (DMEM, Gibco) supplemented with $10 \%$ FBS, penicillin $(50 \mathrm{U} / \mathrm{ml})$, and streptomycin (50 $\mu \mathrm{g} / \mathrm{ml}$ ), and were maintained at $37^{\circ} \mathrm{C}$ with $5 \% \mathrm{CO}_{2}$. To detect the neurite outgrowth, glass coverslips in 24-well plates were pre-coated with $100 \mu \mathrm{g} / \mathrm{ml}$ poly-L-lysine (PLL), washed and dried, and the SH-SY5Y cells were resuspended with DEME in the presence of $100 \mathrm{ng}$ MAG-Fc (R\&D systems) with preimmune sera $(1: 100)$; rat sera immunized with pcDNAGMCSF-NgR-PirB (1:100) or pcDNA3.1(+), pcDNAGMCSF, and pcDNA-NgR-PirB (1:100), or rabbit anti-NgR and anti-PirB (anti-NgR/PirB), and anti-NgR or anti-PirB polyclonal antibody (1:200, positive controls) was then plated separately at a density of $1 \times 10^{6}$ cells $/ \mathrm{ml}$ onto corresponding coverslips pre-coated with immobilized substrates (PLL). The SH-SY5Y cells without MAG-Fc and the SH-SY5Y cells with 100ng MAG-Fc were designed as blank control and negative control, respectively. Cells were cultured 24 hours before fixation with $4 \%$ paraformaldehyde and immunostained with a mouse anti- $\beta$ III tubulin monoclonal antibody (Millipore, USA). Neurite length was quantified by measuring the lengths of individual neurites using the Image-Pro Plus 5.0 image analysis software from Media Cybernetics, from at least ten areas under light microscopy per condition, and from three independent experiments in duplicate wells. Average neurite length was used for statistic analysis.

\section{Spinal Cord Dorsal Hemisection Model}

After the sixth immunization, rats were anesthetized with an intraperitoneal injection of pentobarbital sodium $(40 \mathrm{mg} / \mathrm{kg})$ and body temperature was maintained at $37^{\circ} \mathrm{C}$ during the period of anesthesia with a temperature-controlled heating pad. The dorsal hemisection was performed at a depth of $1.6 \mathrm{~mm}$ from the left dorsal surface of the cord, using a pair of microscissors, to sever the dorsal corticospinal tracts (CST), after exposed by laminectomy at the level of T9-T11. Among a total of 45 rats, 39 rats received spinal cord hemisection, seven rats were immunized with pcDNA-GMCSF-NgR-PirB (GMCSF-NgR-PirB IM), eight rats respectively were immunized with pcDNA3.1(+) (Blank IM), pcDNA-GMCSF (GMCSF IM), and pcDNA-NgR-PirB (NgR-PirB IM), eight rats were unimmunized for negative control (SCI), and six rats were untouched as normal control (Control). Among them, one immunized with pcDNA-GMCSF-NgR-PirB, two respectively immunized with pcDNA3.1(+), pcDNA-GMCSF and pcDNA-NgR-PirB, and two unimmunized rats died during the first week after SCI. Among the remaining 36 rats, six rats of each group underwent the entire experiment procedures.

\section{Assessments of Motor Function}

In order to assess the motor function, long-term behavioral experiments were then carried out using the same injury model. Rat locomotor recovery after SCI was assessed according to the Basso, Beattie, and Bresnahan (BBB) locomotor scale [28]. This scale provides a measure of hindlimb function ranging in a scale from 0 (complete paralysis) to 21 (normal locomotion) by assessing hindlimb joint movements, stepping, trunk position and stability, forelimb-hindlimb coordination, paw placement, toe clearance, and tail position. The rats were observed in an open field and evaluated within a period of 4 minutes. BBB was performed once weekly up to 6 weeks post-injury. Blind scoring ensured that observers were not aware of the treatment received by individual rats.

Locomotion was tested by CatWalk automated gait analysis using the CatWalk system (Noldus Information Technology, Wageningen, The Netherlands) [29]. In brief, as animals traverse a horizontal glass plate, their footfalls are recorded by a video camera positioned beneath the walkway. 
Data files were collected and analyzed using version 10.5 of the CatWalk software. For analysis, crossing speed was standardized, and one uninterrupted crossing of the walkway was required. Individual paw prints were labeled by one observer blinded to the groups. Recordings were taken at 6 weeks post injury. The following parameters were selected for analysis: stride length, base of support (BOS), swing (s) and stance (s). Stride length is the distance between successive placements of the same paw. Base of support (BOS) is the average width between either the front or hind paws. Stance (s) is the duration of the paw in contact with the glass plate during a step cycle. Swing (s) or swing phase is the duration of no contact of a paw with the glass plate in a step cycle.

\section{Anterograde and Retrograde Tracing of Corticospinal Tract (CST)}

All rats were injected with biotinylated-dextran amine (BDA) 4 weeks after injury for CST anterograde tracing. According to a previous study [30], animals were anesthetized as mentioned above and fixed on a stereotaxic apparatus. After shaving and sterilizing, the midline scalp was incised, and the cranium was exposed. Six holes approximately $0.5 \mathrm{~mm}$ in diameter were drilled with a mill on each hemisphere, based on stereotaxic coordinates measured from bregma and calculated using a rat atlas $\left(\mathrm{P}_{1}: 0.8 \mathrm{~mm}, \mathrm{~L}: 2.0 \mathrm{~mm} ; \mathrm{P}_{2}: 1.8 \mathrm{~mm}, \mathrm{~L}: 2.4 \mathrm{~mm} ; \mathrm{P}_{3}: 2.8\right.$ $\mathrm{mm}, \mathrm{L}: 2.7 \mathrm{~mm} ; \mathrm{P}_{4}: 0.8 \mathrm{~mm}, \mathrm{R}: 2.0 \mathrm{~mm} ; \mathrm{P}_{5}: 1.8 \mathrm{~mm}, \mathrm{R}: 2.4$ $\mathrm{mm} ; \mathrm{P}_{6}: 2.8 \mathrm{~mm}, \mathrm{R}: 2.7 \mathrm{~mm}$ ). Then, a microsyringe was fixed on the stereotactic injection support, with the injection points above the holes. BDA (10000MW, $0.3 \mu \mathrm{L}, 10 \%$, Invitrogen, USA) was slowly injected into each hole within 10 minutes at $1.5 \mathrm{~mm}$ depth from the cerebral cortex, and needles were placed for 10 minutes, and then withdrawn slowly.

For the retrograde tracing, 16-18 hours before perfusion six weeks after the dorsal hemisection, a retrograde axonal tracer nuclear yellow (NY, 376.37 MW; 0.5\% in PBS; Sigma) was slowly injected into the left lateral sciatic nerve of the hind limb, as previously described [27, 31]. For each injection, $6 \mu 1$ NY was delivered for a period of 15 minutes via a $10-15 \mathrm{~nm}$ inner diameter glass pipette attached to a Picopump, and needles were placed for 10 minutes.

Four weeks after injection of BDA or 16-18 hours injection of NY, the animals were perfused transcardially with PBS followed by $4 \%$ paraformaldehyde. For anterograde tracing to the lesion site, the spinal cord $3 \mathrm{~mm}$ caudal was sagittally sectioned $30 \mu \mathrm{m}$ using a cryostat (Leica CM1900, Bannockburn, IL), while the spinal cord 4-6 mm rostral for retrograde tracing to the lesion site was transversely sectioned at a thickness of $10 \mu \mathrm{m}$, and both were thaw-mounted on gelatin-coated slides. For anterograde tracing, the sagittal sections caudal to the lesion site were stained with 3,3'-diaminobenzidine (DAB), and then all the pictures were captured at the same area of tissue sections using an optical microscope
(Olympus, Tokyo, Japan). For retrograde tracing, the transverse sections rostral to the lesion site were directly examined, and all pictures were captured at the same area of tissue sections using a ZEISS LSM510 Meta confocal microscope. The staining of BDA-labeled axons and NY-labeled neurons were further quantitated by the mean optical density and fluorescence intensity, respectively, using the Image-Pro Plus 5.0 image analysis software (Media Cybernetics Inc., Atlanta, GA, USA).

\section{Histological Analysis}

After the last functional tests 6 weeks after injury, rats were deeply anesthetized by intraperitoneal injections of sodium pentobarbital $(80 \mathrm{mg} / \mathrm{kg})$ and sacrificed by transcardial perfusion with $100 \mathrm{ml}$ of cold $0.1 \mathrm{M}$ phosphate buffered saline (PBS, pH 7.4) followed by $400 \mathrm{ml}$ of $4 \%$ paraformaldehyde in cold 0.1 M PBS ( $\mathrm{pH}$ 7.4). After perfusion, a 12-mm block of the spinal cord containing the injury site in the middle was carefully removed. Then $6 \mathrm{~mm}$ containing the entire injury site in the middle for longitudinal sections $(20 \mu \mathrm{m})$, and 3-6 $\mathrm{mm}$ rostral and 3-6 $\mathrm{mm}$ caudal to the injury site for transverse sections $(20 \mu \mathrm{m})$ were respectively postfixed in the same fixation solution, dehydrated overnight in ethanol, and embedded in a paraffin block. Serial longitudinal and transverse sections were made. For further morphological and morphometric analyses, two sets of slides, each containing serial sections, were respectively stained with hematoxylin and eosin (HE) and toluidine blue (Nissl), then all images were collected at the same area of tissue sections by an Olympus BX60 microscope. Morphometric analysis of spared spinal cord (SC) parenchyma was carried out in the most central portion of the SC judging from the presence of ependyma in the longitudinal sections of tissue by HE stain. Area measurements were performed using the Image-Pro Plus 5.0 image analysis software (Media Cybernetics Inc., Atlanta, GA, USA). The sections 3-6 $\mathrm{mm}$ rostral to the injury site were analyzed for residual ventral horn motoneurons, and the number of surviving ventral horn neurons were confirmed by the exhibition of Nissl substance, euchromatic nucleus and nucleolus [27]. From each spinal cord, approximately 50 sections were inspected, and all slides were assessed blindly with respect to treatment. For frozen section preparation, the specimen was transferred to a solution containing $30 \%$ sucrose in $0.1 \mathrm{M} \mathrm{PBS}(\mathrm{pH} 7.4)$ at $4^{\circ} \mathrm{C}$, until it stayed at the bottom of the container, then $6 \mathrm{~mm}$ spinal cord segment containing the entire injury site for longitudinal sections, 3-6 $\mathrm{mm}$ rostral and 3-6 $\mathrm{mm}$ caudal to the injury site for transverse sections were embedded in tissue freezing medium (Tissue-Tek, Miles, Elkart, IN); serial $20 \mu \mathrm{m}$ thick cross sections were obtained using a cryostat (Leica CM1900, Bannockburn, IL) and thaw-mounted on gelatin-coated slides. The frozen sections were stored at $4^{\circ} \mathrm{C}$ and would be further used for immunofluorescence staining. 


\section{Immunofluorescence Staining}

The immunofluorescence staining was used to further identify the specificity of the immunoglobulins detected according to the NgR and PirB expression, and the extent of axons regeneration through the expression of growth associated protein43 (GAP-43) in both control and immunized rats. In brief, spinal cord frozen sections were mounted on gelatin-coated slides. After incubation with a blocking buffer (10\% normal goat serum, $0.3 \%$ Triton $\times 100$ in $0.01 \mathrm{M}$ PBS) for 1 hour at room temperature, sections reacted with primary antibodies, including a rabbit anti-NgR polyclonal antibody $(1: 100$, Santa Crue), a mouse anti-PirB monoclonal antibody (1:100, Sino Biological), or a rabbit anti-GAP-43 polyclonal antibody (1:100, Santa Crue) at $4{ }^{\circ} \mathrm{C}$ overnight, and then with FITC conjugated goat anti-rabbit antibody or $\mathrm{Cy} 3$ conjugated goat anti-mouse antibody $\left(1: 100\right.$, Sigma) for 1 hour at $37^{\circ} \mathrm{C}$. After incubation with a second antibody, slides were incubated with DAPI for 5 minutes, cover slipped, and all pictures were captured at the same area of tissue sections using a ZEISS LSM510 Meta confocal microscope. The immunofluorescence staining of NgR, PirB and GAP-43 was further quantitated by the fluorescence intensity using the Image-Pro Plus 5.0 image analysis software (Media Cybernetics Inc., Atlanta, GA, USA).

\section{Statistical Analysis}

All data were presented as mean \pm standard error of mean (SEM). Statistical significance was assessed between groups using a one-way analysis of variance (ANOVA) test and independent-samples $t$ test with the SPSS 19.0 for Windows covariance software package. The level of significance was set at $P<0.05$.

\section{Results}

\section{Preparation of Eukaryotic Expression Vector pCDNA-GMCSF-NgR-PirB}

The choice of polypeptide sequence is important for the therapeutic efficacy of DNA vaccine. In the present study, the total length DNA fragments of human GM-CSF, NgR and PirB were subcloned into eukaryotic expression vector pcDNA3.1(+), and respectively constructed pcDNAGMCSF, pcDNA-NgR-PirB and pcDNA-GMCSF-NgRPirB expressing vectors as a single open reading frame. The diagram of the pcDNA-GMCSF-NgR-PirB vector is shown in Fig. 1a. After identified by PCR (Fig. 1b), restriction endonuclease digestion (Fig. 1c), and DNA sequencing analysis, the recombinant plasmids pcDNA-GMCSF-NgR-PirB were transfected into $\mathrm{CHO}$ cells mediated by lipofectamine, and the expression of GMCSF-NgR-PirB fusion protein was detected after transfected 24 hours by using immunofluorescence (Fig. 1d) and Western blot (Fig. 1e), respectively. The above results showed that the recombinant eukaryotic expression plasmids pcDNA-GMCSF-NgR-PirB were constructed and expressed successfully, which were obtained for immunization in the following studies.

\section{Immunogenicity of GMCSF-NgR-PirB Nucleic Acid Vaccine}

Similar to protein vaccines, the therapeutic efficacy of nucleic acid vaccines was also determined by the immunogenicity of the expressed antigen. In the present study, the specific antibody responses were detected by ELISA. The results showed that high levels of antibodies against NgR (Fig. 2a), and PirB (Fig. 2b), were detected in all rats immunized with pcDNAGMCSF-NgR-PirB and pcDNA-NgR-PirB nucleic acid vaccines, and both were significantly higher than that of unimmunized SCI rats (Fig. 2; two-tailed Student's $t$ test, $* P<0.05$, $* * P<0.01)$. However, in control rats immunized with pcDNA3.1(+), pcDNA-GMCSF and unimmunized rats in control or SCI group, almost no anti-NgR nor anti-PirB antibody was detected. Interestingly, the average antibody titer in rats immunized with pcDNA-GMCSF-NgR-PirB was significantly higher than that immunized with pcDNA-NgR-PirB (Fig. 2; two-tailed Student's $t$ test, ${ }^{\#} P<0.05,{ }^{\# \#} P<0.01$ ), which indicated that GM-CSF effectively enhanced the immunogenicity of $\mathrm{NgR}$ and PirB in GMCSF-NgR-PirB immunized rats.

\section{Promotional Effects of Antisera from GMCSF-NgR-PirB Immunized Rats on Neurite Outgrowth}

To determine if the anti-NgR or anti-PirB antibody in the sera of immunized rats also could neutralize myelin associated inhibitors and promote neurite outgrowth in vitro, preimmune sera, sera from pcDNA3.1(+), pcDNA-GMCSF, pcDNA-NgR-PirB and pcDNA-GMCSF-NgR-PirB immunized rats, and rabbit anti-NgR/PirB, rabbit anti-NgR or PirB polyclonal antibody as positive controls were added separately into the culture medium of SH-SY5Y cells in the presence of MAG, one of the three known myelin-associated inhibitors. The SH-SY5Y cells without MAG and the SH-SY5Y cells with MAG were designed as blank control and negative control, respectively. After cultured 24 hours, neurite lengths were measured according to the neurons immunostained with a mouse anti- $\beta$ III tubulin monoclonal antibody. The results showed that sera from pcDNA-NgR-PirB, pcDNA-GMCSFNgR-PirB immunized rats and anti-NgR/PirB antibody significantly enhanced neurite outgrowth in SH-SY5Y cells as compared with pre-immune sera (Fig. 3a, b; one-way ANOVA, $\mathrm{F}=60.426, P<0.0001)$. The average length of neurites treated 
a

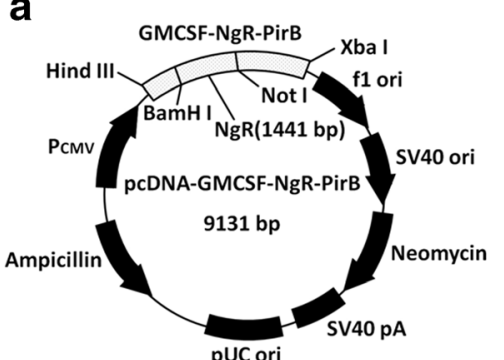

d

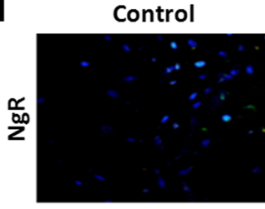

pcDNA3.1(+)
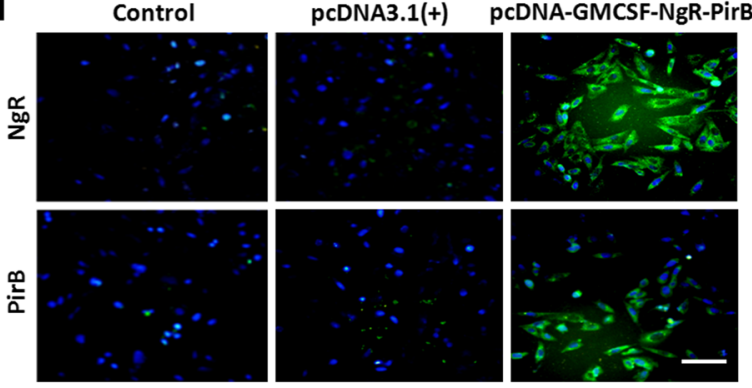

\section{b}

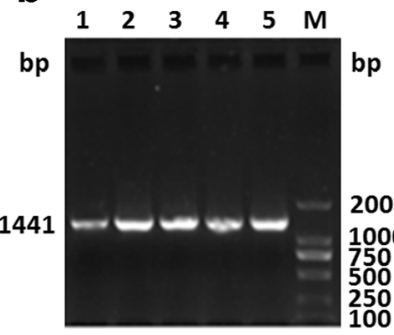

C

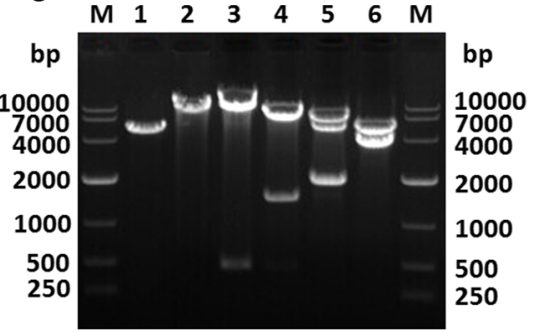

Fig. 1 Preparation of recombinant pcDNA-GMCSF-NgR-PirB eukaryotic expression vector and its expression in CHO cells. (a) Diagram of the constructed pcDNA-GMCSF-NgR-PirB expression vector. (b) Colony PCR identification of recombinant vector of pcDNA-GMCSF-NgRPirB. Lane 1-5: NgR positive PCR products of pcDNA-GMCSF-NgRPirB; M: DNA marker DL2000. (c) Restriction enzymes analysis of recombinant vector of pcDNA-GMCSF-NgR-PirB. M: DNA marker DL10000; Lane 1: pcDNA3.1(+) digested with BamH I; Lane 2: pcDNA-GMCSF-NgR-PirB digested with BamH I; Lane 3: pcDNAGMCSF-NgR-PirB digested with BamH I and Hind III; Lane 4:

pcDNA-GMCSF-NgR-PirB digested with BamH I and Not I; Lane 5: pcDNA-GMCSF-NgR-PirB digested with Not I and Xba I; Lane 6: pcDNA-GMCSF-NgR-PirB digested with Hind III and Xba I. (d) The expression of NgR and PirB in GMCSF-NgR-PirB fusion protein in pcDNA-GMCSF-NgR-PirB transfected CHO cells was detected after transfected 24 hours by using immunofluorescence. Scale bar, $50 \mu \mathrm{m}$. (e) The expression of NgR and PirB in GMCSF-NgR-PirB fusion protein in pcDNA-GMCSF-NgR-PirB transfected $\mathrm{CHO}$ cells was detected after transfected 24 hours by using Western blot

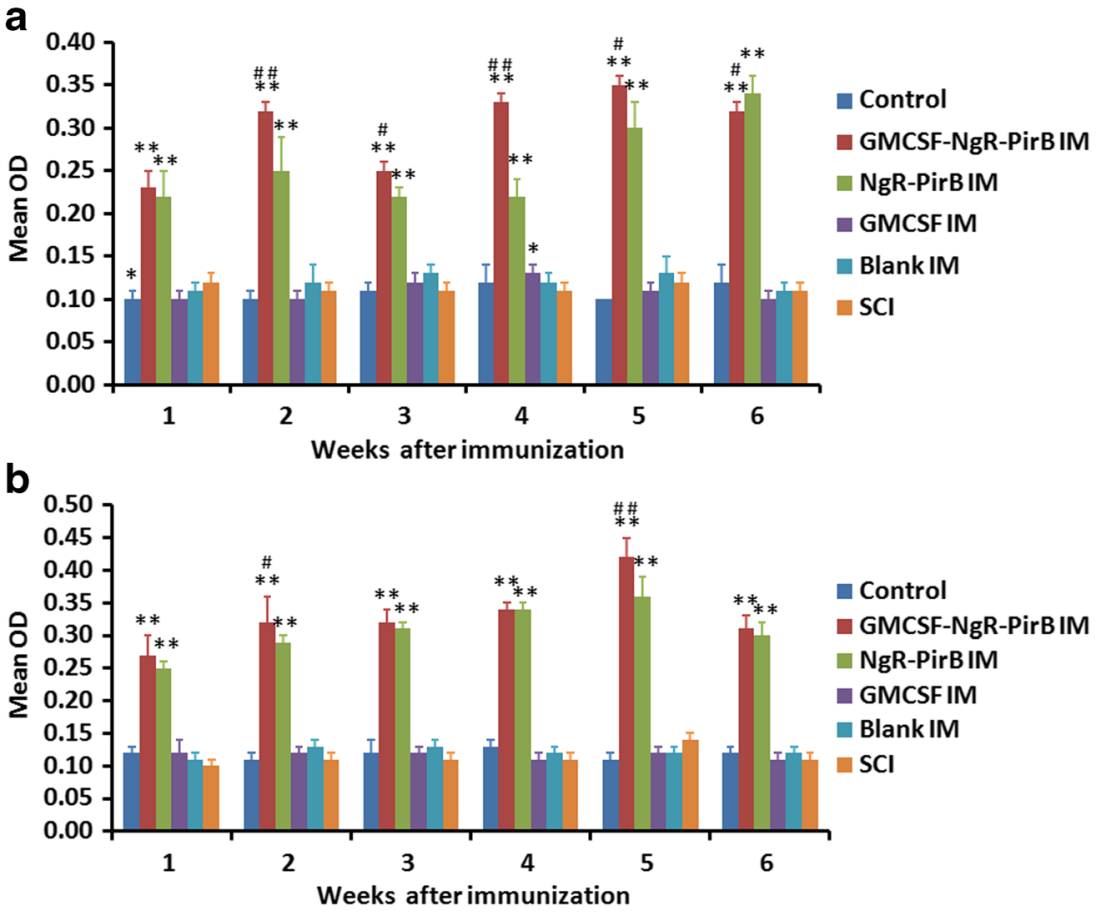

Fig. 2 Detection of antibody response against $\mathrm{NgR}$ and PirB. (a) ELISA detection for specific antibody responses to $\mathrm{NgR}$ in the sera of immunized rats. (b) ELISA detection for specific antibody responses to PirB in the sera of immunized rats. The average antibody titers were shown as mean optical density (OD) at $450 \mathrm{~nm}$ measured with an ELISA reader, and the titer of rats immunized with pcDNA-GMCSF-NgR-PirB as well as
pcDNA-NgR-PirB was significantly higher than that of unimmunized SCI rats, and the average antibody titer in rats immunized with pcDNAGMCSF-NgR-PirB was significantly higher than that immunized with pcDNA-NgR-PirB. $* P<0.05, * * P<0.01$, compared with the unimmunized SCI group; ${ }^{\#} P<0.05,{ }^{\# \#} P<0.01$, compared with NgR-PirB IM group ( $n=6$ per group) 
with sera from GMCSF-NgR-PirB IM rats and anti-NgR/PirB antibody was also significantly increased over that of NgRPirB IM rats (Fig. 3a, b; one way ANOVA, F=60.426, $P<0.0001)$. These results strongly indicated that GMCSFNgR-PirB nucleic acid vaccine could reverse the inhibition of MAG and sera from GMCSF-NgR-PirB IM rats and promoted the neurite outgrowth more significantly than that from NgR-PirB IM rats.

\section{Immunization with GMCSF-NgR-PirB Nucleic Acid Vaccine Promoted Functional Recovery after SCI}

To determine the effect of GMCSF-NgR-PirB nucleic acid vaccine immunization on functional recovery in spinal cord injured rats, besides BBB locomotor, the CatWalk automated gait analysis was also performed. The results of the BBB locomotor showed significant improvements in locomotor recovery in rats immunized with GMCSF-NgR-PirB nucleic acid vaccine starting as early as 1 week, and NgR-PirB IM after 2 weeks, while both lasted up to 6 weeks after SCI as compared to the SCI group (Fig. $4 \mathrm{a} ; P<0.05, P<0.01$ ). Also, significant improvements were found in GMCSF-NgR-PirB
IM rats compared to NgR-PirB IM rats except 1 week and 4 weeks post SCI (Fig. $4 \mathrm{a} ; P<0.05, P<0.01$ ). In the CatWalk automated gait analysis at the sixth week after SCI, the prints of all hind toes in the GMCSF-NgR-PirB nucleic acid vaccine and NgR-PirB immunized rats were very visible, while in the controls they were not clearly separated which indicated signs of toe dragging. The main parameters selected in CatWalk for analysis are marked in Fig. 4b. The stance (s) of left hind paws (LH-stance) and the stride length of left hind paws (LH-stride length) were both significantly increased in the GMCSFNgR-PirB and NgR-PirB vaccinated rats compared with the SCI group (Fig. 4d, e; $P<0.05, P<0.01$ ). Furthermore, the swing ( $\mathrm{s}$ ) of left hind paws (LH-swing), and the base of support (BOS) of hind paws (H-BOS) were all significantly reduced both in the GMCSF-NgR-PirB and NgRPirB vaccinated rats compared with the SCI group (Fig. 4c, f; $P<0.01)$. Importantly, when comparing the GMCSFNgR-PirB IM group with the NgR-PirB IM group, the HBOS was also significantly reduced (Fig. 4f; $P<0.05$ ). The above results suggest that GMCSF-NgR-PirB nucleic acid vaccine noticeably promoted recovery of motor function after SCI, and as a molecular adjuvant, GM-CSF could
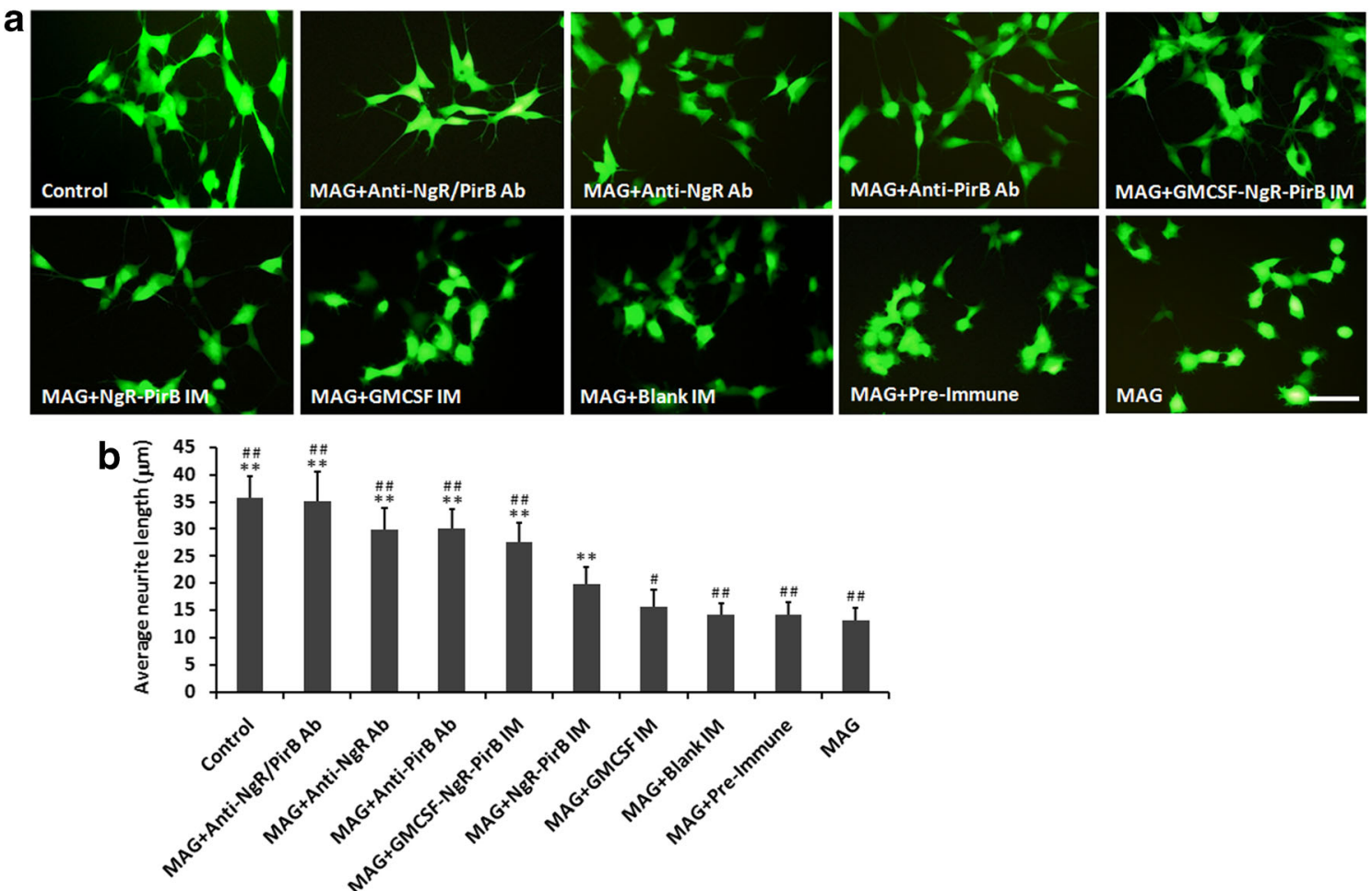

Fig. 3 The inhibitory effect of MAG on neurite outgrowth was reversed by antisera from GMCSF-NgR-PirB immunized rats. (a) Immunostaining with a mouse anti- $\beta$ III tubulin monoclonal antibody for the neurite of SH-SY5Y cells, SH-SY5Y cells cultured with MAG$\mathrm{Fc}$, and SH-SY5Y cells cultured with MAG-Fc in the presence of preimmune sera, sera from pcDNA3.1(+), pcDNA-GMCSF, pcDNA-NgRPirB, and pcDNA-GMCSF-NgR-PirB immunized rats, and rabbit anti$\mathrm{NgR} /$ PirB polyclonal antibody, rabbit anti-NgR or PirB polyclonal antibody, as positive controls. Scale bar, $50 \mu \mathrm{m}$. (b) Quantifications of the average neurite lengths of SH-SY5Y cells. Sera from GMCSF-NgRPirB immunized rats significantly reversed the inhibition of MAG and promoted neurite outgrowth compared with pre-immune sera, as well as sera from GMCSF-NgR-PirB IM rats compared with that from NgR-PirB IM rats. $* * P<0.01$, compared with pre-immune sera group; ${ }^{\#} P<0.05$, ${ }^{\# \#} P<0.01$, compared with NgR-PirB IM group ( $n=6$ per group) 


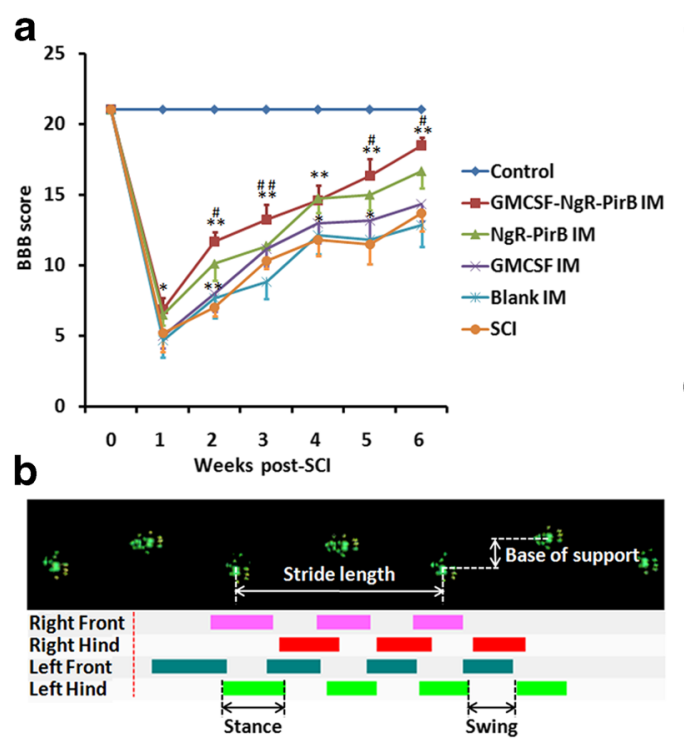

Fig. 4 Vaccination promoted functional recovery after SCI. (a) Except for the normal control, the BBB scores in pcDNA-NgR-PirB and pcDNA-GMCSF-NgR-PirB immunized rats were consistently higher than those in the other negative control groups including pcDNAGMCSF, pcDNA3.1(+) immunized rats, and unimmunized SCI rats, and there was also significant change between the GMCSF-NgR-PirB IM group and the NgR-PirB IM group, except at 1 week and 4 weeks post SCI. (b) Legend of CatWalk. The following parameters were selected for analysis: stride length, base of support (BOS), swing (s), and stance (s). Stride length is the distance between successive placements of the

improve the therapeutic efficacy by enhancing the immunogenicity of $\mathrm{NgR}$ and PirB in GMCSF-NgR-PirB immunized rats.

\section{Vaccination with GMCSF-NgR-PirB Nucleic Acid Vaccine Promoted Axon Regeneration after Spinal Cord Hemisection}

To evaluate whether functional recovery mediated by GMCSF-NgR-PirB nucleic acid vaccine immunization was the consequence of axon regeneration, 6 weeks post SCI, corticospinal tract (CST) anterograde tracing was investigated by $\mathrm{DAB}$ staining of axons through BDA injection in cerebral cortex, and retrograde axonal transport was detected by NYlabeled neurons of spinal cord CST. For anterograde tracing, in longitudinal sections caudal to the lesion site, the number of labeled positive axons and corresponding mean optical intensity in GMCSF-NgR-PirB immunized rats were nearly equally labeled with normal controls, while much more and higher than that in pcDNA-NgR-PirB, pcDNA-GMCSF and pcDNA3.1(+) immunized rats, though there was no significant difference between them. However, when compared with the unimmunized SCI group, the average mean optical intensity of normal controls, GMCSF-NgR-PirB IM and NgR-PirB IM rats was significantly increased (Fig. 5a, c; two-tailed Student's $t$ test, $P<0.01$ ). These results of BDA anterograde tracing suggested that the GMCSF-NgR-PirB nucleic acid
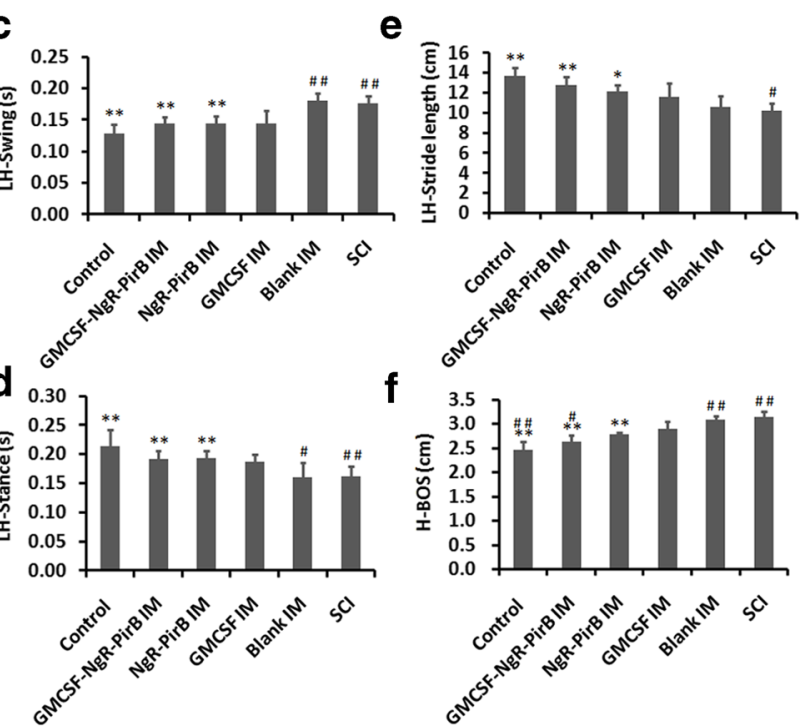

same paw. Base of support (BOS) is the average width between either the front or hind paws. Stance (s) is the duration of the paw in contact with the glass plate during a step cycle. Swing (s) or swing phase is the duration of no contact of a paw with the glass plate in a step cycle. (c) The swing (s) of left hind paws (LH-swing) 6 weeks post SCI. (d) The stance (s) of left hind paws (LH-stance) 6 weeks post SCI. (e) The stride length of left hind paws (LH-stride length) 6 weeks post SCI. (f) The base of support (BOS) of hind paws (H-BOS) 6 weeks post SCI. $* P<0.05$, $* * P<0.01$, compared with unimmunized SCI group; ${ }^{\#} P<0.05,{ }^{\# \#} P<0.01$, compared with $\mathrm{NgR}$ PirB IM group ( $n=6$ per group)

vaccine immunization could efficiently promote axonal outgrowth, repair the fiber contact of injured spinal cord, and the extent of axon regeneration in GMCSF-NgR-PirB immunized rats was better than that in NgR-PirB immunized rats, though there was no significant difference between them.

For retrograde tracing, the result was in accordance with that of anterograde tracing through BDA-labeled axons. In transverse sections rostral to the lesion site, the number of labeled positive neurons and corresponding fluorescence intensity in GMCSF-NgR-PirB immunized rats were nearly equally labeled with normal controls, while much more and higher than that in pcDNA-NgR-PirB, pcDNA-GMCSF and pcDNA3.1(+) immunized rats, though there was no significant difference between them. However, when compared with the SCI group the fluorescence intensity of normal controls, GMCSF-NgR-PirB IM and NgR-PirB IM rats was significantly increased (Fig. 5b, d; two-tailed Student's $t$ test, $P<0.01$ ). These results of NY retrograde tracing suggested that the GMCSF-NgR-PirB nucleic acid vaccine immunization could efficiently promote axonal outgrowth as well as repair the fiber contact of injured spinal cord, and the extent of axon regeneration in GMCSF-NgR-PirB immunized rats was better than that in NgR-PirB immunized rats, though there was no significant difference between them. The results further indicated that, at least to some extent, GM-CSF could enhance the immunogenicity of $\mathrm{NgR}$ and PirB in GMCSF-NgR-PirB immunized rats, and improve the therapeutic efficacy for spinal cord injury. 

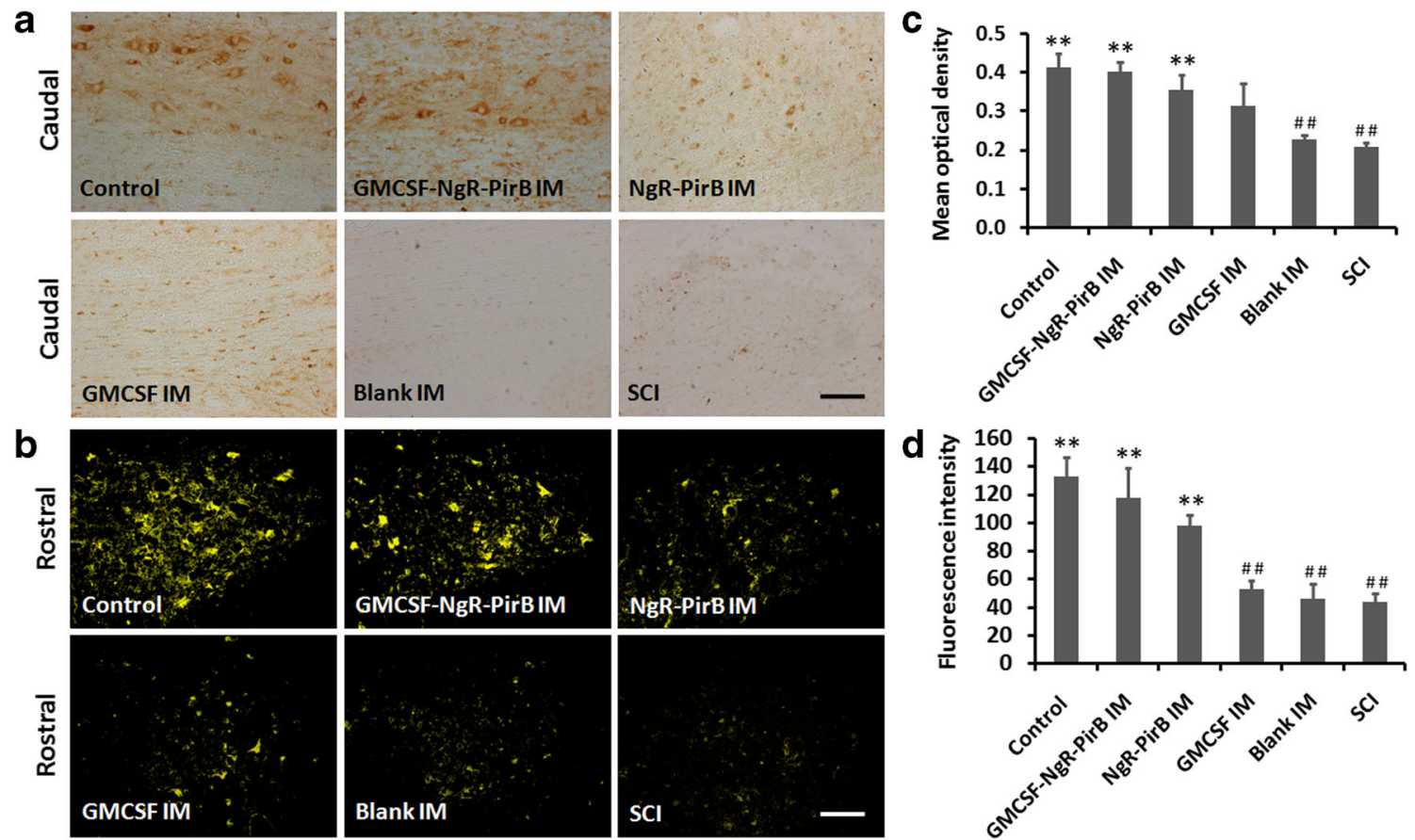

Fig. 5 Vaccination promoted axon regeneration following SCI. (a) Staining of BDA-labeled axons in sagittal sections caudal to the lesion site 6 weeks post SCI. Scale bar, $100 \mu \mathrm{m}$. (b) NY-labeled neurons of transverse sections of the spinal cords at levels rostral to the lesion site 6 weeks post SCI. Scale bar, $100 \mu \mathrm{m}$. (c) Quantifications of mean optical

density of BDA-labeled axons in sagittal sections caudal to the lesion site 6 weeks post SCI. (d) Quantifications of fluorescence intensity in neurons labeled with NY at sagittal sections rostral to the lesion site 6 weeks post SCI. $* * P<0.01$, compared with unimmunized SCI group; ${ }^{\# \#} P<0.01$, compared with the NgR-PirB IM group ( $n=6$ per group)

\section{Immunization of GMCSF-NgR-PirB Nucleic Acid Vaccine Improves Tissue Repair and Neuroprotection after SCI}

To determine whether tissue repair mediated by immunization of GMCSF-NgR-PirB nucleic acid vaccine resulted from the survival of motoneurons in the ventral horn, and the neuroprotective efficacy of the nucleic acid vaccine following SCI, the sections at the sixth week after SCI were stained with HE and Nissl, respectively. In the left portion of the cord, cysts of variable size and shape often trabeculated and foamy macrophages were indicated by HE stain. White and gray substances surrounding the larger cysts, although showing microcysts, were also considered spared tissue. In contrast to GMCSF blank immunized and unimmunized SCI animals, in GMCSF-NgRPirB and NgR-PirB immunized rats, the glial scar surrounding the connective tissue matrix appeared to be less extensive, and there were many longitudinally oriented blood vessels growing into the lesion site and several millimeters distant from the crush site (Fig. 6a). The percentage of spared spinal cord (SC) tissue in the GMCSF-NgR-PirB and NgR-PirB immunized group was significantly smaller than that in the SCI group $(P<0.01)$, similarly in the GMCSF-NgR-PirB IM group compared to the NgR-PirB IM group $(P<0.01)$ (Fig. 6c; two-tailed Student's $t$ test). As shown in Fig. $6 \mathrm{~b}$ by Nissl stain, in the GMCSF-NgRPirB immunizated groups, more residual motoneurons were found in the rostral compared to the lesion sites. Quantitative assessment of numbers of neuronal survival indicated that GMCSF-NgR-PirB and NgR-PirB immunization resulted in a significant enhancement of survival of motoneurons in the ventral horn following SCI, compared to the SCI group (Fig. 6d; two-tailed Student's $t$ test, $P<0.01$, GMCSF-NgR-PirB IM compared with $\mathrm{SCI}$, and $P<0.05$, NgR-PirB IM compared with $\mathrm{SCI})$. The results indicated that immunization of GMCSFNgR-PirB resulted in significantly better tissue repair and neuroprotection than SCI, and GM-CSF could more obviously facilitate the therapeutic efficacy of spinal cord injury through enhancing the immunogenicity of NgR and PirB in GMCSFNgR-PirB immunized rats.

\section{The Expression of NgR, PirB and GAP-43}

To further identify whether the axon regeneration in GMCSFNgR-PirB immunized rats attributed to the neutralization of anti-NgR or anti-PirB antibody induced by GMCSF-NgRPirB nucleic acid vaccine, the expression of NgR, PirB and GAP-43 was detected respectively by immunofluorescence staining, and then was further quantitated by the fluorescence intensity using the Image-Pro Plus 5.0 image analysis software (Media Cybernetics Inc., Atlanta, GA, USA). The results showed that the fluorescence intensity of $\mathrm{NgR}$ and PirB in GMCSF-NgR-PirB immunized rats, and the fluorescence intensity of PirB in normal control rats was much weaker than that of the spinal cord-injured rats (Fig. 7a, b, d; two-tailed 
a

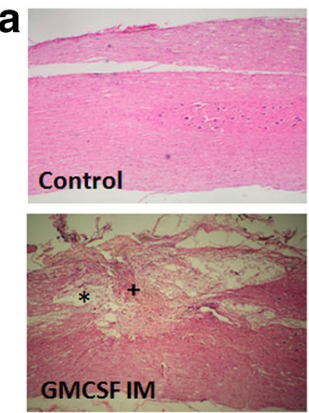

b.
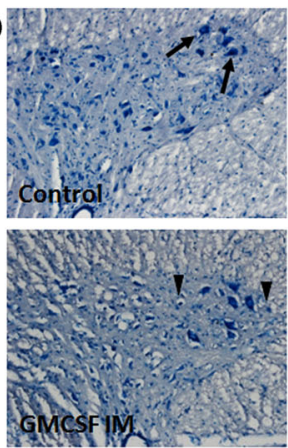
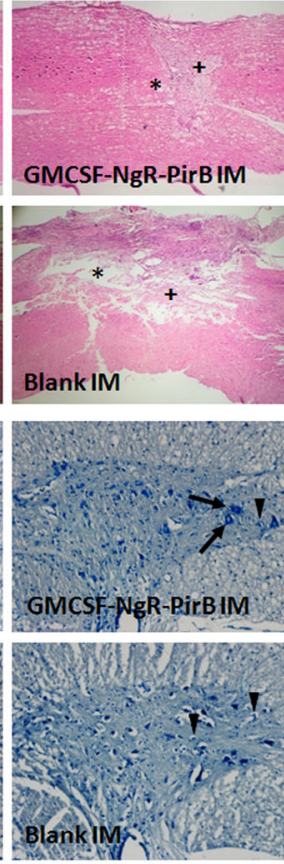
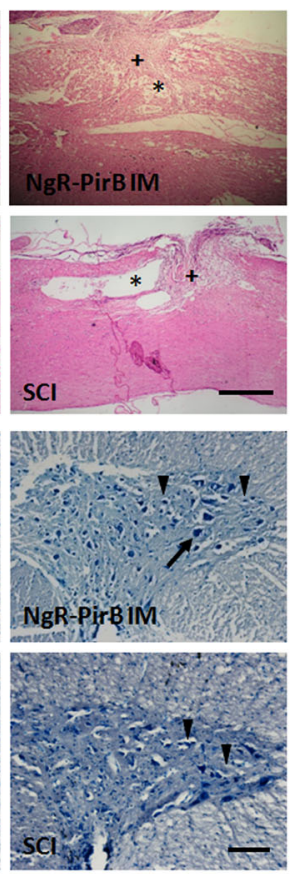

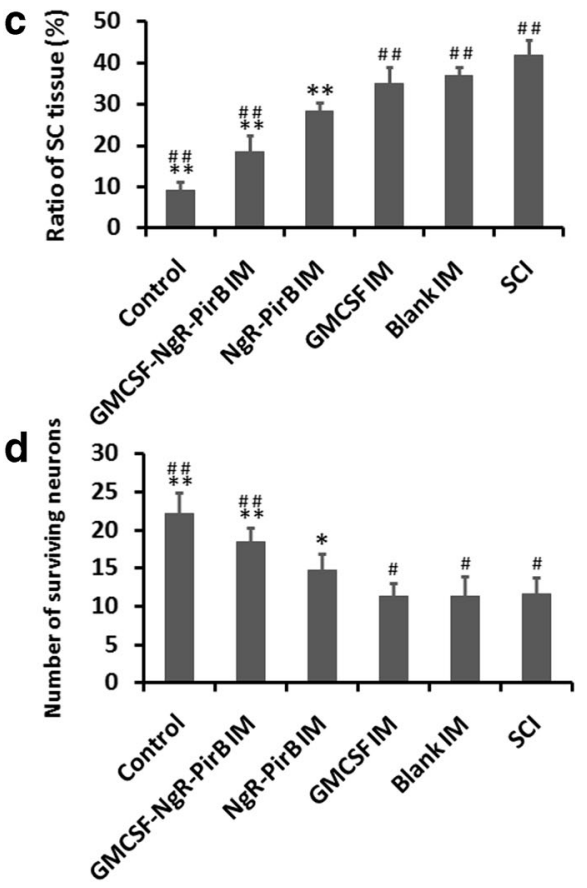

Fig. 6 Histological analyses of spinal cord sections after SCI. (a) HE staining of the sections of spinal cord segment containing the rostrocaudal extension of the lesion cavity. Trabeculated cysts $(*)$ in the left portion of the cord containing foamy macrophages; spared nervous tissue with microcysts (+). Scale bar, $100 \mu \mathrm{m}$. (b) The transverse sections 3-6 $\mathrm{mm}$ rostral to the injury site were analyzed for residual ventral horn motoneurons by Nissl stain. In the pcDNA-GMCSF-NgR-PirB immunized group, the majority of neurons presented undisturbed morphology (arrows), and some in the pcDNA-NgR-PirB immunized group.

Student's $t$ test, $P<0.01$ ), which is consistent with the upregulation of PirB in the injured spinal cord reported by Nakamura and colleagues [16]. However, the fluorescence intensity of anti-GAP-43 staining in GMCSF-NgR-PirB immunized rats was much stronger than that of the SCI rats (Fig. 7c, d; twotailed Student's $t$ test, $P<0.01)$. Furthermore, GMCSF-NgRPirB IM rats also had weaker anti-NgR and anti-PirB, while stronger anti-GAP-43 fluorescence intensity than NgR-PirB IM rats (Fig. 7a-d; two-tailed Student's $t$ test, $P<0.05$, $P<0.01)$. As a crucial marker of neurites growth, GAP-43 is important to show the presence of the regenerating axons in the damaged CNS. The results indicated that the expression of GAP-43 was increased in GMCSF-NgR-PirB immunized rats, and the results in Figs. 5, 6 and 7 were attributed to the enhanced axonal regeneration and functional recovery in GMCSF-NgR-PirB immunized rats after spinal cord injury. These results further confirmed that both $\mathrm{NgR}$ and PirB were upregulated and inhibited regeneration in the injured spinal cord, and suggested that after GMCSF-NgR-PirB immunization, most of the $\mathrm{NgR}$ and PirB molecules were blocked by GMCSF-NgR-PirB-induced anti-NgR and anti-PirB antibody in the spinal cord following injury for 6 weeks. The results also indicated that GM-CSF, as a molecular adjuvant, could improve the immunogenicity of NgR and PirB in GMCSF-
However, transverse sections taken from the SCI group were associated with the presence of ischemic neurons (arrowheads) in ventral horn rostral to the lesion site as well as the GMCSF and Blank IM group. Scale bar, $100 \mu \mathrm{m}$. (c) Quantifications for the percentage of spared spinal cord tissue in different groups by HE stain. (d) Quantifications for the number of surviving ventral horn neurons in spinal cord tissue of different groups by Nissl stain. $* P<0.05, * * P<0.01$, compared with SCI; ${ }^{*} P<0.05$, ${ }^{\# \#} P<0.01$, compared with NgR-PirB IM group ( $n=6$ per group)

NgR-PirB immunized rats and promote axon regeneration more effectively than in the NgR-PirB IM group.

\section{Discussion}

Growing studies have shown that $\mathrm{NgR}$ and PirB, two common receptors for the three well-characterized MAIs, Nogo-A, MAG and OMgp, play crucial roles in the failure of axonal regeneration after SCI. As a result, inhibition of the $\mathrm{NgR}$ and PirB mediated MAIs signal transduction pathway may be an effective therapy strategy for spinal cord injury. In previous studies, some NgR antagonist peptides, such as NEP1-40 [32, 33], a soluble $\mathrm{NgR}[34,35]$, as well as transgenic inhibition of $\mathrm{NgR}$ with a soluble functional blocking $\mathrm{NgR}$ fragment $\mathrm{NgR} 310$ [36], have been proven to promote axonal regeneration and functional recovery after SCI through antagonizing the binding of myelin associated inhibitors to $\mathrm{NgR}$. Other studies also indicated that a monoclonal antibody against $\mathrm{NgR}$ could block the binding of the three MAIs to NgR and reverse the inhibition of neurite outgrowth by CNS myelin in vitro $[34,35]$. With the concept of protective autoimmunity formulated [18], growing immunotherapy strategies promoting axon regeneration and functional recovery after SCI were 

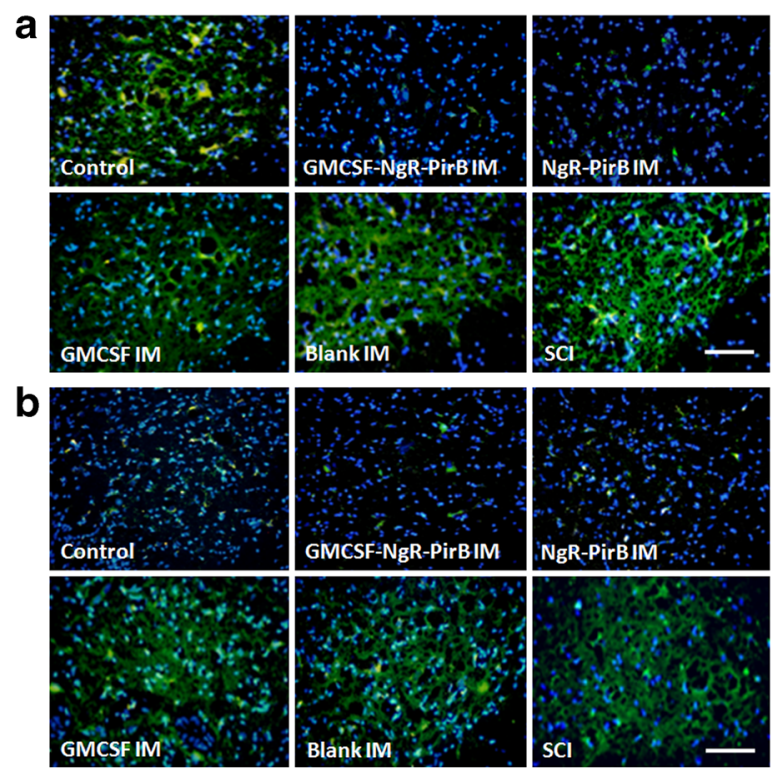

Fig. 7 Anti-NgR antibody, anti-PirB antibody and anti-GAP-43 antibody immunoreactivity in the injured spinal cords of control, immunized, and SCI rats. (a) Anti-NgR antibody immunoreactivity. Scale bar, $100 \mu \mathrm{m}$. (b) Anti-PirB antibody immunoreactivity. Scale bar, $100 \mu \mathrm{m}$. (c) AntiGAP-43 antibody immunoreactivity. Scale bar, $100 \mu \mathrm{m}$. Sagittal sections of the spinal cord showed that the $\mathrm{NgR}(\mathbf{a})$ and $\mathrm{PirB}(\mathbf{b})$ immunoreactivity of GMCSF-NgR-PirB nucleic acid vaccine immunized rats was lower, and the expression of GAP-43 (c) in GMCSF-NgR-PirB nucleic acid

initially achieved [37, 38]. To overcome the inhibitory activity mediated by $\mathrm{NgR}$ in vivo, the protein and DNA vaccines against $\mathrm{NgR}$ were reported respectively, which could to some extent promote functional recovery after SCI in adult rats [23, $24,27]$. However, the therapy effect was not prospective, and in NgR gene knockout mice after spinal cord transection, the 5-HT axons showed no promoting effect on regeneration [39]. Thus, besides $\mathrm{NgR}$, there may be also other factors involved in inhibiting signal transduction which play an important role in axonal regeneration in the injured CNS.

Indeed, besides $\mathrm{NgR}$, PirB, also known as LILRB2, was recently discovered as another common receptor for various MAIs and has also been proposed to mediate nearly all MAIsmediated axon growth inhibition [13]. And the same as $\mathrm{NgR}$, PirB provides a new therapeutic target for treatment of SCI through promoting axonal regeneration and functional recovery [13-16]. PirB gene knockout produced an axonal regeneration effect to a certain extent even better than $\mathrm{NgR}$, showing that in the myelin inhibition, PirB function may be better than that of previously found $\mathrm{NgR}$. If $\mathrm{NgR}$ and PirB are knocked down at the same time, neuronal growth cone collapse induced by myelin can be completely reversed. Experiments in vitro also showed that NgR gene knockout can promote PirB gene knockout to reverse the inhibitory effect of myelin on axon regeneration [17]. The above research confirmed that, in addition to $\mathrm{NgR}$, PirB is also an important receptor that mediated the inhibition of axonal

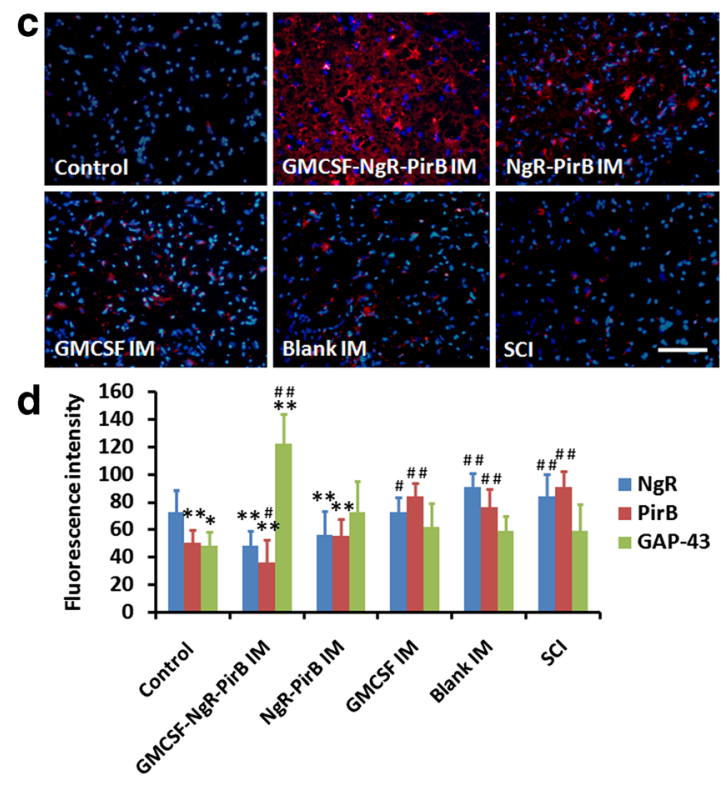

vaccine immunized rats was much higher than that of the spinal cordinjured rats sacrificed at 6 weeks after SCI. The same change was found in the GMCSF-NgR-PirB IM group compared with the NgR-PirB IM group $($ a, b, c). (d) Quantifications of NgR, PirB and GAP-43 immunoreactivity in control, immunized, and SCI rats. $* P<0.05$, $* * P<0.01$, compared with the SCI group; ${ }^{\#} P<0.05,{ }^{\# \#} P<0.01$, compared with the NgR-PirB IM group ( $n=6$ per group)

regeneration of MAIs, suggesting that the treatment of spinal cord injury should focus on the synergistic effect of $\mathrm{NgR}$ and PirB in a future study [40].

Based on the above studies, the aim of this study was to design a novel double targeted GMCSF-NgR-PirB nucleic acid vaccine and induce injured animals' humoral immunity after immunization so that they could produce higher titers of both anti-NgR and anti-PirB antibodies leading to even better functional recovery, while avoiding undesired side effects as much as possible. For this purpose, the present study firstly constructed a recombinant plasmid, pcDNA-GMCSF-NgRPirB encoding human NgR, PirB and GM-CSF, an effective adjuvant molecular, and formed a composite nucleic acid vaccine with liposome. Then, the effects of the antisera on neurite outgrowth of SH-SY5Y cells, axonal regeneration and functional recovery in spinal cord-injured rats were further investigated. The present study may pave the way to further research of the regeneration of the central nervous system (CNS) on the target of NgR and PirB.

To evaluate the efficacy of the double targeted GMCSFNgR-PirB nucleic acid vaccine immunization, a dorsal hemisection model was used for studying axon regeneration, tissue sparing and functional recovery in vivo. The immunohistochemical staining of GAP-43, BDA and NY tracing in the dorsal hemisection model showed that, in most of the GMCSF-NgR-PirB immunized rats, injured CST axons could grow beyond the lesion site into the caudal and rostral spinal 
cord as compared to the lack of such growth in the SCI rats, and, to some extent, the efficacy of GMCSF-NgR-PirB immunized rats was higher than that of NgR-PirB immunized rats, though there was no significant difference between them. This was likely a result of the induction of anti- $\mathrm{NgR}$ and antiPirB antibodies after immunization that reversed some inhibitory activities of MAIs and, therefore, promoted axonal regeneration of the CST. Furthermore, in the present study, immunization with GMCSF-NgR-PirB induced significantly better morphological and functional recovery compared with NgR-PirB in spinal cord injured rats. These findings suggest that, in addition to stimulating axon regeneration, the GMCSF-NgR-PirB nucleic acid vaccine may also have a neuroprotective effect which may contribute to the overall functional recovery after SCI. However, the mechanism underlying such neuroprotection remains to be investigated. Interestingly, GM-CSF as a molecular adjuvant could improve the immunogenicity of NgR and PirB in GMCSF-NgR-PirB immunized rats and improve the therapeutic efficacy for spinal cord injury.

\section{Conclusions}

A novel double targeted GMCSF-NgR-PirB nucleic acid vaccine was prepared in this study and the vaccine inoculation could stimulate higher production of anti-NgR and anti-PirB antibody than that immunized with NgR-PirB nucleic acid vaccine. The production of antibodies against $\mathrm{NgR}$ and PirB could block the inhibitory effects mediated by MAG, as well as promote nerve regeneration and functional recovery after spinal cord injury. GM-CSF, as a molecular adjuvant could improve the immunogenicity of NgR and PirB in GMCSF-NgR-PirB immunized rats, and promoted axon regeneration more effectively. Therefore, our present study may provide a new immunotherapy strategy for treatment of the injured spinal cord, as well as the other CNS, such as brain and optic nerve. This strategy may therefore be used in translating this treatment into a clinically applicable therapy in the future. However, the mechanism underlying such neuroprotection remains to be further investigated.

Acknowledgments This study was supported by the National Natural Science Foundation of China (81772064 and 81671903), the Open Project of the State Key Laboratory of Trauma, Burn and Combined Injury, Third Military Medical University (SKLKF201706), the Innovative Foundation for Postgraduates of Chongqing (CYS18313), and the Undergraduate Training Programs for Innovation and Entrepreneurship of Chongqing University of Technology (2018CX035).

Required Author Forms Disclosure forms provided by the authors are available with the online version of this article.

Publisher's Note Springer Nature remains neutral with regard to jurisdictional claims in published maps and institutional affiliations.

\section{References}

1. Jazayeri SB, Beygi S, Shokraneh F, Hagen EM, Rahimi-Movaghar $\mathrm{V}$. Incidence of traumatic spinal cord injury worldwide: a systematic review. Eur Spine J 2015; 24: 905-918.

2. Chiu WT, Lin HC, Lam C, Chu SF, Chiang YH, Tsai SH. Review paper: epidemiology of traumatic spinal cord injury: comparisons between developed and developing countries. Asia Pac J Public Health 2010; 22: 9-18.

3. van den Berg ME, Castellote JM, Mahillo-Fernandez I, de PedroCuesta J. Incidence of spinal cord injury worldwide: a systematic review. Neuroepidemiology 2010; 34: 184-192.

4. Krause JS, Devivo MJ, Jackson AB. Health status, community integration, and economic risk factors for mortality after spinal cord injury. Arch Phys Med Rehabil 2004; 85: 1764-1773.

5. Brösamle C, Halpern ME. Nogo-Nogo receptor signaling in PNS axon outgrowth and pathfinding. Mol Cell Neurosci 2009; 40: 401409.

6. Chen MS, Huber AB, van der Haar ME, et al. Nogo-A is a myelinassociated neurite outgrowth inhibitor and an antigen for monoclonal antibody IN-1. Nature 2000; 403: 434-439.

7. GrandPré T, Nakamura F, Vartanian T, Strittmatter SM. Identification of the Nogo inhibitor of axon regeneration as a Reticulon protein. Nature 2000; 403: 439-444.

8. Prinjha R, Moore SE, Vinson M, et al. Inhibitor of neurite outgrowth in humans. Nature 2000; 403: 383-384.

9. McKerracher L, David S, Jackson DL, Kottis V, Dunn RJ, Braun PE. Identification of myelin-associated glycoprotein as a major myelinderived inhibitor of neurite growth. Neuron 1994; 13: 805-811.

10. Mukhopadhyay G, Doherty P, Walsh FS, Crocker PR, Filbin MT. A novel role for myelin-associated glycoprotein as an inhibitor of axonal regeneration. Neuron 1994; 13: 757-767.

11. Kottis V, Thibault P, Mikol D, et al. Oligodendrocyte-myelin glycoprotein (OMgp) is an inhibitor of neurite outgrowth. J Neurochem 2002; 82: 1566-1569.

12. Wang YT, Lu XM, Zhu F, et al. Ameliorative effects of p75NTRED-Fc on axonal regeneration and functional recovery in spinal cord-injured rats. Mol Neurobiol 2015; 52: 1821-1834.

13. Atwal JK, Pinkston-Gosse J, Syken J, et al. PirB is a functional receptor for myelin inhibitors of axonal regeneration. Science 2008; 322: 967-970.

14. Matsushita H, Endo S, Kobayashi E, et al. Differential but competitive binding of Nogo protein and class i major histocompatibility complex (MHCI) to the PIR-B ectodomain provides an inhibition of cells. J Biol Chem 2011; 286: 25739-25747.

15. Filbin MT. PirB, a second receptor for the myelin inhibitors of axonal regeneration Nogo66, MAG, and OMgp: Implications for regeneration in vivo. Neuron 2008; 60: 740-742.

16. Nakamura Y, Fujita Y, Ueno M, Takai T, Yamashita T. Paired immunoglobulin-like receptor B knockout does not enhance axonal regeneration or locomotor recovery after spinal cord injury. J Biol Chem 2011; 286: 1876-1883.

17. Omoto S, Ueno M, Mochio S, Takai T, Yamashita T. Genetic deletion of paired immunoglobulin-like receptor $\mathrm{B}$ does not promote axonal plasticity or functional recovery after traumatic brain injury. J Neurol Sci 2010; 30: 13045-13052.

18. Schwartz M, Moalem G, Leibowitz-Amit R, Cohen IR. Innate and adaptive immune responses can be beneficial for CNS repair. Trends Neurosci 1999; 22: 295-299.

19. Hauben E, Butovsky O, Nevo U, et al. Passive or active immunization with myelin basic protein promotes recovery from spinal cord contusion. J Neurosci 2000; 20: 6421-6430.

20. Kipnis J, Yoles E, Schori H, Hauben E, Shaked I, Schwartz M. Neuronal survival after CNS insult is determined by a generically encoded autoimmune response. J Neurosci 2001; 21: 4564-4571. 
21. Yoles E, Hauben E, Dalgi O, et al. Protective autoimmunity is a physiological response to CNS trauma. J Neurosci 2001; 21: 3740-3748.

22. Cohen IR, Schwartz M. Autoimmune maintenance and neuroprotection of the central nervous system. J Neuroimmunol 1999; 100: 111-114.

23. Yu P, Huang L, Zou J, et al. DNA vaccine against NgR promotes functional recovery after spinal cord injury in adult rats. Brain Res 2007; 1147: 66-76.

24. Yu P, Huang L, Zou J, et al. Immunization with recombinant Nogo66 receptor $(\mathrm{NgR})$ promotes axonal regeneration and recovery of function after spinal cord injury in rats. Neurobiol Dis 2008; 32 : 535-542.

25. Liu L, Zhu L, Zou Y, et al. Panax notoginseng saponins promotes stroke recovery by influencing expression of Nogo-A, NgR and p75NGF, in vitro and in vivo. Biol Pharm Bull, 2014, 37(4):560-568.

26. Shi $\mathrm{X}, \mathrm{Yu} \mathrm{W}$, Yang $\mathrm{T}$, et al. Panax notoginseng saponins provide neuroprotection by regulating NgR1/RhoA/ROCK2 pathway expression, in vitro and in vivo. J Ethnopharmacol, 2016, 190: 301-312.

27. Wang YT, Lu XM, Zhu F, et al. The use of a gold nanoparticlebased adjuvant to improve the therapeutic efficacy of $\mathrm{hNgR}-\mathrm{Fc}$ protein immunization in spinal cord-injured rats. Biomaterials 2011; 32: 7988-7998.

28. Basso DM, Beattie MS, Bresnahan JC. A sensitive and reliable locomoter ranting scale for open field testing in rats. J Neurotrauma 1995; 12: 1-21.

29. Hamers FP, Koopmans GC, Joosten EA. Catwalk-assisted gait analysis in the assessment of spinal cord injury. J Neurotrauma 2006; 23: 537-548.

30. Hendriks WT, Eggers R, Ruitenberg MJ, et al. Profound differences in spontaneous long term functional recovery after defined spinal tract lesions in the rat. J Neurotrauma 2006; 23:18-35.

31. Decherchi P, Gauthier P. Regrowth of acute and chronic injured spinal pathways within supra-lesional post-traumatic nerve grafts. Neuroscience 2000; 101: 197-210.
32. GrandPre T, Li S, Strittmatter SM. Nogo-66 receptor antagonist peptide promotes axonal regeneration. Nature 2002; 417: 547-551.

33. Li S, Strittmatter SM. Delayed systemic Nogo-66 receptor antagonist promotes recovery from spinal cord injury. J Neurosci 2003; 23: 4219-4227.

34. Li S, Liu BP, Budel S, et al. Blockade of Nogo-66, myelinassociated glycoprotein, and oligodendrocytemyelin glycoprotein by soluble Nogo-66 receptor promotes axonal sprouting and recovery after spinal injury. J Neurosci 2004; 24: 10511-10520.

35. Li W, Walus L, Rabacchi SA, et al. A neutralizing anti-Nogo66 receptor monoclonal antibody reverses inhibition of neurite outgrowth by central nervous system myelin. J Biol Chem 2004; 279: 43780-43788.

36. Li S, Kim JE, Budel S, Hampton TG, Strittmatter SM. Transgenic inhibition of Nogo-66 receptor function allows axonal sprouting and improved locomotion after spinal injury. Mol Cell Neurosci 2005; 29: 26-39.

37. Huang DW, McKerracher L, Braun PE, David S. A therapeutic vaccine approach to stimulate axon regeneration in the adult mammalian spinal cord. Neuron 1999; 24: 639-647.

38. Wang YT, Lu XM, Chen KT, Shu YH, Qiu CH. Immunotherapy strategies for spinal cord injury. Curr Pharm Biotechnol 2015; 16 : 492-505.

39. Wills ZP, Mandel-Brehm C, Mardinly AR, McCord AE, Giger RJ, Greenberg ME. The nogo receptor family restricts synapse number in the developing hippocampus. Neuron 2012; 73: 466-481.

40. Lu XM, Wei JX, Xiao L, Shu YH, Wang YT. Experimental and clinical advances in immunotherapy strategies for spinal cord injury target on MAIs and their receptors. Curr Pharm Design 2016; 22: 728-737. 